\title{
The Preparation of Chicken Ex Ovo Embryos and Chorioallantoic Membrane Vessels as In Vivo Model for Contrast-Enhanced Ultrasound Imaging and Microbubble- Mediated Drug Delivery Studies
}

\author{
Bram Meijlink ${ }^{1}$, Ilya Skachkov ${ }^{1}$, Antonius F. W. van der Steen ${ }^{1,2}$, Nico de Jong ${ }^{1,2}$, Klazina Kooiman ${ }^{1}$ \\ ${ }^{1}$ Department of Biomedical Engineering, Thoraxcenter, Erasmus MC University Medical Center Rotterdam ${ }^{2}$ Laboratory of Acoustical Wavefield Imaging, \\ Faculty of Applied Sciences, Delft University of Technology
}

\section{Corresponding Authors}

Bram Meijlink

k.meijlink@erasmusmc.n

Klazina Kooiman

k.kooiman@erasmusmc.nl

\section{Citation}

Meijlink, B., Skachkov, I., van

der Steen, A.F.W., de Jong, N.,

Kooiman, K. The Preparation of Chicken

Ex Ovo Embryos and Chorioallantoic

Membrane Vessels as In Vivo Model for Contrast-Enhanced Ultrasound Imaging and Microbubble-Mediated Drug Delivery Studies. J. Vis. Exp. (168), e62076, doi:10.3791/62076 (2021).

\section{Date Published}

February 9, 2021

DOI

$10.3791 / 62076$

URL

jove.com/video/62076

\section{Abstract}

The chicken embryo and the blood-vessel rich chorioallantoic membrane (CAM) is a valuable in vivo model to investigate biomedical processes, new ultrasound pulsing schemes, or novel transducers for contrast-enhanced ultrasound imaging and microbubble-mediated drug delivery. The reasons for this are the accessibility of the embryo and vessel network of the CAM as well as the low costs of the model. An important step to get access to the embryo and CAM vessels is to take the egg content out of the eggshell. In this protocol, three methods for taking the content out of the eggshell between day 5 and 8 of incubation are described thus allowing the embryos to develop inside the eggshell up to these days. The described methods only require simple tools and equipment and yield a higher survival success rate of $90 \%$ for 5 -day, $75 \%$ for 6 -day, $50 \%$ for 7 -day, and $60 \%$ for 8 -day old incubated eggs in comparison to ex ovo cultured embryos ( $50 \%)$. The protocol also describes how to inject cavitation nuclei, such as microbubbles, into the CAM vascular system, how to separate the membrane containing the embryo and CAM from the rest of the egg content for optically transparent studies, and how to use the chicken embryo and CAM in a variety of short-term ultrasound experiments. The in vivo chicken embryo and CAM model is extremely relevant to investigate novel imaging protocols, ultrasound contrast agents, and ultrasound pulsing schemes for contrast-enhanced ultrasound imaging, and to unravel the mechanisms of ultrasound-mediated drug delivery.

\section{Introduction}

Ex ovo chicken embryos and the blood-vessel rich chorioallantoic membrane (CAM) have proven to be a suitable model to investigate various biological and biomedical processes like embryogenesis, oncology, and 
drug delivery ${ }^{1,2,3,4}$. Ultrasound has been used for imaging of the embryonic heart development ${ }^{4,5}$ and for activating cavitation nuclei upon injection, such as microbubbles, for vascular drug delivery ${ }^{6,7}$. Chicken embryos are inexpensive, require less infrastructure and equipment, and have less strict legislation compared to other animal models ${ }^{8}$. The chicken embryo and CAM vessels are easily accessible after opening the egg whereas this proves to be much more difficult with mammalian embryos and vessels. Besides this, the chicken embryo and CAM vessels provide a heartbeat and a pulsating blood flow. The CAM shows similarities in vessel anatomy with mammals and can be used for drug screening $8,9,10$. Because of these characteristics, the CAM vessels have also proven to be a suitable model to investigate contrast enhanced ultrasound imaging (CEUS) ${ }^{11,12,13,14,15,16}$. In addition, the model can be used to optically investigate the behavior of ultrasound contrast agents in an ultrasound field using an ultra-high-speed camera and the effect of acoustic radiation force on propelling, binding and extravasation of drugs $^{7,17}, 18,19$. Although the chicken embryo and CAM are less suitable for long term experiments, they can be beneficial for short term in vivo experiments.

To increase visibility and controllability over the chicken embryo and CAM during experiments, it is important to take the egg content containing the embryo and CAM out of the eggshell ${ }^{18}$. Previous chicken embryo studies involving ultrasound contrast agents used 5 to 6-day old embryos ${ }^{7,11,12,17,19}$ and 14 to 18-day old embryos ${ }^{13,14,15,16}$. Multiple approaches have been described in detail to take the egg content out of the shell ${ }^{18,20,21}$. However, to the best of our knowledge, the previously published approaches focus on taking the egg content out of the eggshell after 3 days of incubation (i.e., Hamburger \& Hamilton $(\mathrm{HH})$ stage $19-20^{22}$ ), and continue the culture ex ovo. This ex ovo culture approach has multiple disadvantages including increased risk of fatalities during culture $(\sim 50 \%)^{1,18}$, the use of antibiotics ${ }^{18,20}$, and decreased total vessel length in comparison to in ovo growth $^{23}$. Since culturing the embryo within the eggshell is providing the most natural environment, it is easiest to incubate the embryo within the eggshell until the day of the experiment. For this reason, an approach in which the egg content is taken out of the eggshell at 5 to 8 days of incubation would be beneficial especially for experiments on 5 to 8-day old embryos.

In this protocol, we describe three methods to take the egg content out of the eggshell when the embryo is at day 5

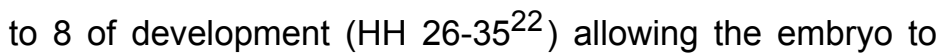
develop within the eggshell until the day of the experiment. The CAM vessel size ranges from 10-15 $\mu \mathrm{m}$ in diameter, in the smaller capillaries of an 8-day old embryo ${ }^{24}$, to $115-136$ $\mu \mathrm{m}$ in diameter in the larger vessel of 6 and 8-day old embryos $^{24,25}$. The three described methods only require basic lab tools and reduce the risk of complications before the experiment has begun, thereby reducing unnecessary costs and labor. We also detail a method to separate the membrane containing the embryo and CAM from the yolk sack making the CAM optically transparent for microscopy studies. Because the membrane containing the embryo and CAM can be pinned down on for example a holder with an acoustic membrane, the setup can also be made acoustically transparent ${ }^{26}$, allowing the combination of microscopy and ultrasound studies when the light path will be affected by the yolk. Finally, we describe several other ultrasound setups that can be used for ultrasound or CEUS imaging. 


\section{Protocol}

All animal experiments were conducted in accordance with the Netherlands Experiments on Animals Act and in accordance with the European Council (2010/63/EU) on the protection of animal use for scientific purposes.

\section{Embryo preparation protocol}

1. Incubation of the fertilized chicken eggs

1. Store freshly fertilized chicken eggs at $15^{\circ} \mathrm{C}$ for up to one week.

2. To incubate the fertilized eggs, place them vertically with the pointy side down into a $37{ }^{\circ} \mathrm{C}$, humidified incubator. Turning the eggs during incubation is not necessary.

NOTE: Write the starting date of incubation of top of the egg using a permanent marker.

2. Preparing up to 5-day (120 h) old embryo's (HH stage $26-28)^{22}$

1. Preparation of working area

1. Warm up a heating plate to $37^{\circ} \mathrm{C}$.

2. Place a metal egg holder (Figure 1A,B), a metal weighing boat holder (Figure 1C,D), and a 10 $\mathrm{mL}$ Erlenmeyer filled with PBS on the heating plate.

3. Fill a weighing boat $(85 \mathrm{~mm} \times 85 \mathrm{~mm} \times 25 \mathrm{~mm})$ with a $10 \mathrm{~mm}$ layer of ultrasound gel and place the filled weighing boat in the prewarmed metal weighing boat holder.

NOTE: Filling the weighing boat with ultrasound gel will raise the embryo and CAM. This can be beneficial for the injection or imaging of the embryo and CAM but is not necessary for taking the embryo and CAM out of the eggshell.

4. Prepare a few pieces of tape (around $3 \mathrm{~cm}$ length) with part of one end folded back on itself so that does not stick anymore.

2. Taking the egg content out of the eggshell

1. Take a 5-day old incubated fertilized egg and transfer it to the prewarmed metal egg holder (Figure 1A,B). Make sure to keep the egg in the same orientation (i.e., date on top).

NOTE: It is important to keep the egg in the same orientation to keep the air sac and the embryo and CAM at the same position in the top of the egg.

2. Use the pointy back of a tweezer (or similar; Figure $1 \mathrm{E}$ ) to make a small indent on the very top of the egg (where the date is written) (Figure 2A).

3. Use the pointy back of the tweezers to make a second indent on the side of the egg around $2 / 3$ down the egg (Figure 2B).

NOTE: Be careful not to make the indent too big and create a hole. If by accident a hole is created, seal the hole with tape and do not make another indent.

4. Using the larger tweezers (Figure 1E), take out a small piece of eggshell from the indented area on top of the egg (with written date). Make sure the air sac in the top of the eggshell makes contact with the air outside the egg, but do not penetrate the shell too deep.

NOTE: If the shell is penetrated too deep when making the top indent, the embryo and CAM 
could be damaged and the embryo will not survive the removal from the shell. It is important that the small hole on the top creates air contact between the inside and outside of the egg. If this is not done, a vacuum will be created in the next steps of the procedure which will result in large air bubbles getting trapped under the CAM making the embryo and CAM useless. To check the position of the air sac inside the egg, a light source can be used since its position is not always exactly at the top and can also be more to the side.

5. Use a $5 \mathrm{~mL}$ syringe and $19 \mathrm{G}$ needle to penetrate the shell through the second indent on the side 2/3 down the egg and withdraw $\sim 2$ $\mathrm{mL}$ of egg white (Figure $2 \mathrm{C}$ ).

NOTE: Make sure the needle is pointing down towards the bottom of the egg to prevent the chance of damaging the embryo and CAM. This step creates a larger air pocket in the top of the egg needed for removal of the egg content. If a hole is accidentally created instead of an indent in step 1.2.2.3, puncture the tape with the needle for the withdrawal of the egg white. Reseal the puncture with another piece of tape.

6. Take out the needle and use tape to seal the gap on the side (Figure 2D).

NOTE: To prevent egg white from leaking out of the egg, the top hole can be closed using a finger before taking the needle out. If egg white keeps leaking out with the tape already in place, first remove the egg white with a piece of tissue to make sure the tape sticks properly.
7. Empty the syringe by adding the egg white to the weighing boat.

8. Use the large tweezers (Figure 1E) to enlarge the small opening on the top of the egg (Figure 2E). When looking inside the egg through the opening on the top, the embryo and CAM are visible. Keep locating the embryo and CAM while taking away as much of the eggshell as possible (Figure 2F) .

NOTE: Keep on moving the egg to maintain maximum visibility on the position of the embryo and CAM inside the shell. Make sure the edge of the opening in the shell does not go lower than the CAM. Besides this, do not penetrate the inner membrane and prevent sharp edges.

9. After creating the opening, turn the egg $180^{\circ}$ and place the egg back in the egg holder in such a way that the created opening at the top of the egg is now facing towards the bottom. The embryo will float up and will become invisible from the bottom (Figure 2G) which takes 1-2 min. Make sure the whole embryo and CAM (including all the vessels) have disappeared and only yolk is visible before proceeding to the next step (Figure 2H).

NOTE: If the embryo is still visible from the bottom after $2 \mathrm{~min}$, turn the egg clockwise for 1-2 min. This will help the embryo and CAM to float up.

10. Remove the tape from the side opening. Look whether the inside of the egg now bulges out of the bottom opening. If this is the case, proceed to the next step. If not, use the needle on the 
syringe to puncture the opening on the side once more to release the vacuum in the egg. Make sure to point upwards with the needle to prevent the chance of puncturing the yolk sack. Continue until the egg bulges out of the bottom opening.

11. While holding the bottom of the egg close to the weighing boat in the metal weighing boat holder (Figure 1C,D), gently but quickly make a horizontal scratch in the membrane over the entire width of the opening using one of the sharp points of the small tweezer (Figure 1F) and gently drop the egg content into the weighing boat (Figure 2I).

NOTE: If the egg content does not come out, use the needle on the syringe to puncture the side opening again with the needle pointing upwards.

12. If the embryo is in the weighing boat sideways, it will usually go up by itself. If this does not happen, use a piece of tissue paper to reposition the embryo. Put one side of the tissue paper on the embryo, drag the tissue paper to the other end, and release the tissue paper with a few drops of $\sim 30 \mu \mathrm{L}$ of $\operatorname{PBS}\left(37^{\circ} \mathrm{C}\right)$ using a plastic Pasteur pipet.

13. Visually check whether the embryo is alive by ensuring the heartbeat is still present, the CAM vessels are intact and there is no bleeding, and there is no leakage of yolk. If one of these things is not correct, discard the embryo and CAM because it will not be viable.
14. Make sure the embryo and CAM are kept at 37 ${ }^{\circ} \mathrm{C}$ and do not dry out because this will make the CAM vessels deteriorate and eventually the embryo will die. To prevent this, regularly put small drops of $\sim 30 \mu \mathrm{L}$ of $37{ }^{\circ} \mathrm{C}$ PBS on the embryo and CAM.

3. Preparing 6 to 7-day (144-168 h) old embryo's (HH stage $28-32)^{22}$

1. Preparation of working area

1. Prepare the stage as described in section 1.2.1.

2. Taking the egg content out of the eggshell

1. Two hours in advance of the experiment, take a 6 to 7-day old incubated egg and rotate the egg $180^{\circ}$ inside the incubator so that the top of the egg is facing towards the bottom. After $1 \mathrm{~h}$, rotate the egg back to its original position and leave for another $1 \mathrm{~h}$.

NOTE: Rotating the egg $2 \mathrm{~h}$ in advance of the experiment will make it easier to take the egg content out of the shell.

2. After rotating, take the egg from the incubator.

3. Perform step 1.2.2.2 until step 1.2.2.4.

4. Use a $5 \mathrm{~mL}$ syringe and $19 \mathrm{G}$ needle to penetrate the shell through the second indent on the side $2 / 3$ down the egg and withdraw between 5-6 mL of egg white. Make sure the needle is pointing down towards the bottom of the egg.

NOTE: With the $5 \mathrm{~mL}$ syringe we used, it is possible to withdraw up to $6 \mathrm{~mL}$ so only one penetration is needed. 
5. Take out the needle and use a piece of tape to seal the gap on the side (Figure 2D).

6. Empty the syringe by adding the egg white to the ultrasound gel in the weighing boat.

7. Use the large tweezers (Figure 1E) to enlarge the small opening on the top of the egg (Figure 2E). Try to make the opening as big as possible but make sure the edge of the opening in the shell does not go lower than the CAM. Besides this, do not penetrate the inner membrane and try to prevent sharp edges.

8. Fill a syringe with $\sim 1 \mathrm{~mL}$ more of $37^{\circ} \mathrm{C}$ PBS than the withdrawn volume during step 1.3.2.4.

9. Take off the tape from the side gap, penetrate the gap with the filled syringe, and empty it into the shell. Make sure the needle is pointing down towards the bottom of the egg.

NOTE: Since egg white has a higher viscosity $(\sim 160 \mathrm{cP})^{27}$ than PBS $(\sim 1 \mathrm{cP})$, substituting the egg white with PBS reduces both tension and stress on the embryo and CAM while taking the egg content out of the shell.

10. Take out the needle and quickly reseal the gap with a piece of tape (Figure 2D).

11. Turn the egg $180^{\circ}$ and place the egg back in the egg holder in such a way that the created opening at the top of the egg is now facing towards the bottom. Turn the egg clockwise until the whole embryo and CAM (including all the vessels) have disappeared and only yolk is visible.

12. Perform step 1.2.2.10 until step 1.2.2.14.
4. Preparing 8-day (192 h) old embryo's (HH stage $32-35)^{22}$

1. Preparation of working area

1. Warm up a heating plate to $37^{\circ} \mathrm{C}$.

2. Place a metal weighing boat holder (Figure 1C,D) and a $10 \mathrm{~mL}$ Erlenmeyer filled with PBS on the heating plate.

3. Take a shallow container of $170 \times 110 \times 70 \mathrm{~mm}$, or similar, and fill the container with $1 \mathrm{~L}$ of 37 ${ }^{\circ} \mathrm{C}$ PBS.

4. Place a weighing boat $(85 \times 85 \times 25 \mathrm{~mm})$ in a 90 $\mathrm{mm}$ diameter Petri dish. Place the Petri dish and the weighing boat at the bottom of the container and make sure they are completely submerged.

2. Taking the egg content out of the eggshell

1. Two hours in advance of the experiment, take an 8-day old incubated egg and rotate the egg $180^{\circ}$ inside the incubator so that the top of the egg is facing towards the bottom. After $1 \mathrm{~h}$, rotate the egg back to its original position and leave for another $1 \mathrm{~h}$.

NOTE: Rotating the egg $2 \mathrm{~h}$ in advance of the experiment will make it easier to take the egg content out of the shell.

2. Take an 8-day old incubated egg from the incubator.

3. Hold the egg horizontally and use the pointy back of the large tweezer (Figure 1E) to make a small indent $1 / 2$ down the egg. Continue making small indents in a ring pattern $360^{\circ}$ round the eggshell. Use a spacing of $\sim 10 \mathrm{~mm}$ between the indents. 
NOTE: During this procedure, small cracks can start to form between the indents.

4. After creating the small indents all around the shell, make one bigger hole by cracking the shell between two small indents using the pointy back of the large tweezer.

5. Completely submerge the egg in the $37^{\circ} \mathrm{C}$ PBS and keep it submerged for $5 \mathrm{~min}$. After $5 \mathrm{~min}$, keep the egg close to the weighing boat inside the container. Put the top of both thumbs in the large hole and gently open the egg. The egg will crack along the small indents.

6. When the crack is formed all the way around the eggshell, gently try to pull the two eggshell pieces apart and keep on gently moving the two pieces back and forth until the egg content is separated from the shell. Then, gently drop the egg content into the weighing boat.

NOTE: By moving the two pieces of eggshell back and forth, more PBS will flow into the eggshell, which will help in separating the egg content from the shell. Sometimes, a bit of egg white will stick to the inside of the eggshell. When this happens, use the tweezers to separate the egg white from to shell.

7. Slowly raise the Petri dish containing the weighing boat and egg content from the PBS. When out of the PBS, slightly tilt the weighing boat to remove the excess PBS.

8. Place the weighing boat containing the egg content into the metal weighing boat holder and move to the desired experimental setup.

\section{Selected Applications}

1. Injecting microbubbles and/or other solutions into the CAM vessels

1. Preparing the injection setup

1. Pull glass needles from glass capillary tubes using a micro forge (Figure $\mathbf{3 A}$ ) or buy pulled glass capillary needles.

2. In case the tip of the glass capillary needle is not beveled, break off a small part of the tip of the needle. Fill the glass needle with mineral oil and place it in a micro-injection system. Make sure there are no air bubbles in the mineral oil in the glass needle.

NOTE: The mineral oil is added as per instruction of the manufacturer of the injection system we used.

3. Empty the pulled-glass capillary needle as far as the micro-injection system allows and partly refill the glass needle with air.

NOTE: The small bit of air will prevent the mixture of the mineral oil and the to-be-injected solution.

4. Put $10 \mu \mathrm{L}$ of the desired solution, in this protocol microbubbles, on a piece of waxy film (Figure 3B). If more than one solution is needed, solutions can be mixed before pipetting ${ }^{7}$.

NOTE: Before filling the needle with microbubbles, leave the microbubble drop on the waxy film for $\sim 1$ min so that the microbubbles will float to the top of the drop and get concentrated. For the F-type custom-made ultrasound contrast agent ${ }^{28}$, 
this concentration step will increase the tobe-injected microbubble concentration with $\sim 30 \%$. The post-injection concentration in the chicken embryo blood will be between $32 \mathrm{x}$ $10^{3}$ microbubbles/ $\mu \mathrm{L}$ for 5 -day old embryos and $19 \times 10^{3}$ microbubbles/ $\mu \mathrm{L}$ for 6 -day old embryos.

5. Fill the glass needle with the microbubble and/or other solution by positioning the glass needle tip into the drop on the waxy film. When aspirating microbubbles, make sure to position the needle tip in the top of the liquid drop to aspirate the microbubble enriched solution.

NOTE: Before injecting microbubbles, raise the tip of the glass needle to its highest point and wait for $\sim 2 \mathrm{~min}$. This will ensure the microbubbles will concentrate into the tip of the glass needle.

2. Injection into the CAM vessels

1. Before injection, look at the CAM under a stereo microscope and select the best vessel to inject. Always inject into one of the veins of the embryo. These are the vessels in which the blood flow moves towards the embryo. Veins are lighter in color than the arteries due to the oxygenated blood $^{29}$. In addition, veins are always on top of the artery with two exceptions namely the anterior and posterior vitelline veins (i.e., the less branched veins, indicated with asterisks in Figure 6A,B) which do not have an artery in their surroundings.

NOTE: Injecting into one of the branches will limit the obstruction of the blood flow during injection. Good injections sites have been indicated with arrowheads in Figure 6A,B. It is crucial to inject into the vein, since this will force the injected substance to flow towards the embryo. Besides this, injecting into the artery will result in a massive bleeding when removing the glass needle which will kill the embryo.

2. Position the glass needle and the embryo in such a way that the glass needle tip and the selected vein are in the same focal plane and in the same line of direction. Try to position the needle as horizontal as possible parallel to the selected vein. The needle tip should touch the vessel wall.

NOTE: By positioning the glass needle as horizontal as possible, the chance of piercing through the entire vessel is lower.

3. After positioning, slowly advance and penetrate the vessel wall with the glass needle. During penetration, the CAM will first be pushed away by the movement of the glass needle. Keep advancing the glass needle until the vessel wall is penetrated. NOTE: If by accident the vessel is pierced through and through, slowly retract the needle to get back into the lumen. When back inside the lumen, slightly lift the needle up and move forward along the vessel to reposition the needle.

4. After penetration, slightly retract the glass needle to better position the tip inside the vessel lumen and move the glass needle sideways to check it is not attached to the vessel wall. Slowly inject a small amount of the solution to confirm that the tip is positioned inside the vessel lumen (Figure $3 \mathbf{C}$ ).

5. Make sure the injected solution follows the blood flow. If it does not, slightly move the glass needle and 
keep injecting small amounts until the glass needle is positioned correctly ${ }^{17}$.

6. When the desired amount is injected, leave the glass needle in the vessel for $\sim 15 \mathrm{~s}$ to prevent a massive bleeding. Then, move the glass needle a little bit sideways, up and down, and back and forth a few times to allow a gently retraction of the glass needle. NOTE: Some bleeding is normal. For every injection use a new glass needle because the glass needle gets easily clogged with and blunt from egg white.

3. Microscopy imaging of the embryo and/or CAM vessels

1. Preparing holder with acoustic membrane

1. Take a cell culture chamber consisting of a square plastic holder with two parallel 50 $\mu \mathrm{m}$ thick acoustically transparent polycarbonate membranes ${ }^{26}$, further referred to as holder with acoustic membrane. Close both ports with a lid.

2. Use a scalpel to remove one of the two membranes from the holder with acoustic membrane.

NOTE: To remove the membrane, cut the membrane just next to the glue line on the plastic. Be careful not to slip off from the edge to prevent damage to the other membrane.

3. Prepare $\sim 15 \mathrm{~mL}$ of $2 \%$ agarose in demi water solution by heating to between $80-95{ }^{\circ} \mathrm{C}$ in a small glass beaker. Cool the glass beaker with the dissolved agarose solution under a running cold-water tap.

NOTE: If the agarose is too hot it will melt the acoustic membrane which will create an uneven surface.
4. When the solution is cooled down to around $37^{\circ} \mathrm{C}$, slowly pour the solution in the holder with acoustic membrane until it fills up the entire holder. Slightly tilt the holder with acoustic membrane so the agarose layer will distribute evenly inside the plastic frame (Figure 4A). Make sure the agarose layer is flat and let the agarose set at room temperature.

2. Removing embryo and CAM from yolk sack and placing on holder with acoustic membrane

1. Take out the egg content out of the egg as described in section $1.2,1.3$, or 1.4 .

2. If needed, inject the CAM with microbubbles and/or other solution(s) as described in section 2.1.2.

3. Fill a $1 \mathrm{~L}$ Petri dish with $\sim 500 \mathrm{~mL}$ of $37{ }^{\circ} \mathrm{C}$ PBS and place the holder with acoustic membrane with agarose on the bottom of the dish. Make sure the agarose layer is facing up.

4. Use small scissors to quickly cut into the membrane of the yolk sack, also called Vitellus membrane, around the entire CAM while the egg content is in the weighing boat (Figure 4B). Keep the scissors in the same position and rotate the weighing boat while cutting for better precision and more speed.

NOTE: From the moment the first cut is made, the yolk will start to leak. This reduces the visibility of the embryo and CAM. Try to cut all the way around the CAM within 6-7 cuts. This should not take much longer than $20 \mathrm{~s}$. The small tweezers (Figure 1F) can be used to 
hold the edge of vitelline membrane and prevent cutting into the CAM.

5. Use a tablespoon to scoop up the cut-out membrane containing the embryo and CAM from the weighing boat. Slowly raise the spoon from the weighing boat and visually inspect whether the cut-out membrane containing the embryo and CAM are still attached to the remainder yolk sack membrane. When this is the case, use the scissors to make an extra cut. While scooping, slightly tilt the spoon to get rid of as much yolk as possible but do not let it dry out. Transfer the cut-out membrane containing the embryo and CAM to the $1 \mathrm{~L}$ Petri dish, submerge in the $37^{\circ} \mathrm{C}$ PBS, and remove the spoon.

6. When the membrane containing the embryo and CAM is submerged in the $37^{\circ} \mathrm{C}$ PBS, use the small tweezers (Figure 1F) to grab one edge of the membrane and gently swirl around the membrane to get rid of the yolk that is still attached.

7. When all the yolk is removed, use the small tweezers to move the membrane containing the embryo and CAM and position it above the holder with acoustic membrane.

8. Use one insect specimen pin to pin down the membrane containing the embryo and CAM in one corner. Avoid piercing the vessels in the CAM and only pin down the membrane.

9. Use a second insect specimen pin to pin down the membrane containing the embryo and CAM on the diagonally opposite corner.
10. Slowly lift the holder with the acoustic membrane containing the embryo and CAM from the $37^{\circ} \mathrm{C}$ PBS. Slightly tilt the holder to get rid of most of the PBS.

11. Use the small tweezers (Figure 1F) to stretch and evenly distribute the membrane containing the embryo and CAM over the holder with acoustic membrane and pin down the rest of the membrane. Make sure the membrane containing the embryo and CAM is slightly stretched to assure it is flat (Figure 4C).

12. Place the holder with acoustic membrane with the pinned down membrane containing the embryo and CAM into a microscopy setup which is kept at $37^{\circ} \mathrm{C}$.

13. Place a coverslip or an acoustically and optically transparent membrane (depending on the desired objective and use of ultrasound or not) on top of the region of interest on the embryo or CAM (Figure 4D) to allow optical visualization.

4. Ultrasound imaging of the chicken embryo and/or CAM vessels

1. Ultrasound imaging from the side of the chicken embryo and CAM vessels

1. Take out the egg content as described in section 1.2, 1.3, or 1.4. However, do not use a standard weighing boat. Instead, use a custom-made weighing boat with one acoustically transparent wall.

NOTE: The standard weighing boat was adjusted by cutting off one side of the weighing 
boat and replacing it with a window of polyester foil which was glued together using epoxy glue.

2. Submerge the preferred ultrasound transducer in a $37^{\circ} \mathrm{C}$ water bath and position in the desired spot with the required standoff distance.

3. Place the weighing boat in the water bath in such a way that the transparent wall is facing the transducer. Make sure the weighing boat is deep enough to be level with the transducer but avoid water getting into the weighing boat (Figure 5A).

4. If desired, add another setup to the top of the embryo or CAM vessels, like a microscope or a laser (Figure 5A).

2. Ultrasound imaging from the top of the embryo and CAM vessels without acoustic interference

1. Fill a $2 \mathrm{~L}$ beaker glass with $37^{\circ} \mathrm{C}$ PBS. Place a $500 \mathrm{~mL}$ beaker glass upside down on the bottom of the $2 \mathrm{~L}$ beaker glass. Avoid air inside the $500 \mathrm{~mL}$ beaker glass.

NOTE: The $500 \mathrm{~mL}$ beaker glass is meant to raise the weighing boat containing the egg content closer to the PBS surface. By substituting the beaker for objects with other sizes, the distance between the transducer and the egg content can be varied.

2. Place the filled $2 \mathrm{~L}$ beaker glass with the $500 \mathrm{~mL}$ beaker glass inside in a $37^{\circ} \mathrm{C}$ water bath.

3. Take out the egg content as described in section $1.2,1.3$, or 1.4 .

4. Wet the egg content with $37^{\circ} \mathrm{C}$ PBS and cover the embryo with clear cling film. This can be done to keep the embryo in the same position and prevent it from rotating or floating away.

NOTE: By wetting the egg content with PBS, it will become less sticky which makes it easier to cover the egg content with clear cling film.

5. Place the weighing boat with the egg content in a $90 \mathrm{~mm}$ diameter Petri dish and slowly submerge the Petri dish in the PBS (Figure 5B). NOTE: Using two clamps on the sides of the Petri dish opposite to one another makes it easier to submerge the Petri dish.

6. Position the ultrasound transducer with the desired standoff distance.

3. Ultrasound imaging of the chicken embryo and CAM vessels with a movable transducer

1. Take out the egg content as described in section $1.2,1.3$, or 1.4

2. Prepare a $2 \%$ agarose solution in demi water by heating the solution up to between $80-95^{\circ} \mathrm{C}$ in a small glass beaker. Cool the glass beaker with the dissolved agarose solution under a running cold water tab.

3. Pour the agarose solution into a flat container to create an about $1 \mathrm{~mm}$ thick agarose pad. When completely cooled down and set, cut the agarose pad to the desired size using a scalpel. NOTE: The thickness of the agarose pad can be changed to obtain the desired focal distance necessary for correct functioning of the ultrasound transducer.

4. Place the agarose pad on top of the embryo and CAM (Figure 5C). Add a few drops of $\sim 30 \mu \mathrm{L}$ of $37^{\circ} \mathrm{C}$ PBS onto the top of the agarose pad 
to create a thin PBS layer between the agarose pad and the transducer.

NOTE: Using PBS will prevent the transducer from sticking to the agarose pad. This is beneficial when, for example, using a motor to mechanically move a two-dimensional transducer to make a three-dimensional scan (Figure 9B $)^{11}$. When the transducer does not need to be moved, the PBS can also be substituted with ultrasound gel.

5. Position the desired ultrasound transducer.

\section{Representative Results}

In this protocol, we describe three methods to take the egg content out of the shell at day 5-8 of incubation ( $\left.\mathrm{HH} 26-35^{22}\right)$. Figure 6 shows the egg content in weighing boats after it was taken out of the shell. The 5-day old embryo and CAM (Figure 6A) was taken out using the method described in section 1.2. The 6 and 7-day old embryos and CAM (Figure 6B,C) were taken out using the method described in section 1.3. The 8-day old embryo and CAM (Figure 6D) was taken out using the method described in section 1.4. No bleeding or damage to the embryo or CAM can be observed, indicating that these methods can be used to safely get the egg content out of the shell without harming the embryo or the CAM vessels. When executed correctly, the method for the 5-day old embryos will provide a viable embryo and intact CAM in $90 \%$ of all procedures. The viability rate is based on the total number of fertilized eggs successfully extracted from the eggshell. With the second method, for 6 and 7-day incubated eggs, the chance of a viable embryo and intact CAM is around $75 \%$ for 6 -day old and around $50 \%$ for 7 -day old. With the third method described for 8day old embryos, the chance of a viable embryo and intact
CAM is around $60 \%$. Differences in developmental stages between the 5 and 8-day old embryos can be observed which concurs with Hamburger and Hamilton ${ }^{22}$. Both the size of the embryo and the complexity of the CAM vessels increase during development (Figure 6A-D). Figure 6C shows a thin patch of agarose on top of the egg content that allows the embryo and CAM to be imaged using the ultrasound setup shown in Figure 5C. After the egg content is taken out of the shell the heartbeat of the embryo is visible with the naked eye. The heart rate of these ex ovo embryos is similar to in ovo embryos at 183 beats per minute (bpm) at day 5 up to $\sim 208 \mathrm{bpm}$ at day $8^{30}$. When kept humidified and at 37 ${ }^{\circ} \mathrm{C}$, the embryo will maintain this heart rate for $\sim 5 \mathrm{~h}$ in the experimental ultrasound setups.

Multiple complications can occur during the previously described three methods. Figure 7A shows trapped air under the CAM which makes the embryo unsuitable for ultrasound imaging and the pressure of the air bubble(s) can also damage the embryo and/or CAM. This problem arises when the air sac inside the shell does not make contact with the air outside the shell when taking the egg content out of the shell. Figure 7B shows a small leakage of yolk from the yolk sack in the top right of the image. This can occur while taking the egg content out of the shell when the yolk sack gets damaged by sharp edges of the shell or when the yolk sack is penetrated by the tweezers. Leakage of the yolk can affect the visibility of the embryo and the CAM vessels. Figure $7 \mathrm{C}$ shows an embryo in which an air bubble is trapped under the CAM. This sometimes occurs in the embryonic development. Another complication which can occur is damage to the vessels. This damage can be created while taking the egg content out of the shell or when performing an injection (Figure 7D). Besides this, the embryo and vessels can also dry out over time (Figure 7E). This occurs when the egg content is not 
sprinkled with PBS. The drying out of the embryo can result in massive capillary obstructions (Figure 7F) which affects the viability of the embryo. The massive capillary obstructions can also occur during development or when the heartbeat of the embryo is not stable.

After the egg content is taken out of the shell without any complication, the embryo can be injected with, for example, ultrasound contrast agents such as microbubbles (Figure 3C). Figure 8 shows circulating microbubbles in the lumen of the blood vessel upon injection. These microbubbles are carried along with the blood flow and stay present in the blood circulation for several hours (Supplemental Video 1). The presence of these microbubbles in the circulation creates the possibility to perform different types of CEUS and drug delivery experiments ${ }^{7,11,12}$. The CAM is ideal to investigate novel ultrasound contrast detection methods for which we show three examples. Figure 9A shows high frequency ultrasound subharmonic imaging of a 6-day old chicken embryo in B-Mode and CEUS before and after microbubble injection. Here, the CAM vessels were injected with $5 \mu \mathrm{L}$ of ultrasound contrast agent and imaging was performed with a preclinical animal ultrasound machine with a MS250 probe (30 MHz transmit and $15 \mathrm{MHz}$ receive frequency, $10 \%$ power). Before microbubble injection, contrast can already be seen inside the embryonic heart in the B-Mode images (Figure 9A-I). This phenomenon is due to the presence of a nucleus in the avian red blood cell which increases the contrast of blood in ultrasound imaging 5,31 . The addition of the microbubbles increased the contrast and the visibility of the embryo, both in the B-Mode and CEUS imaging. Figure 9B shows an optical and a high frequency $3 D$ subharmonic image of a 6-day old embryo and the surrounding vessels. The CAM was injected with 5 $\mu \mathrm{L}$ of ultrasound contrast agent and imaging was performed with a preclinical animal ultrasound machine with MS550s probe (transmission frequency of $40 \mathrm{MHz}$, peak negative pressure $\sim 300 \mathrm{kPa}$ ). These results show that the CEUS imaging combined with a contrast agent can also be used to create high frequency 3D subharmonic images and to image the blood vessels outside the embryo. Figure 9C shows an optical image and an ultraharmonic intravascular ultrasound (IVUS) image made with a custom probe of CAM microvessels of a 6-day old embryo (26 MHz transmit and 39 and $65 \mathrm{MHz}$ receive frequency). CAM vessels were injected with $4 \pm 1 \mu \mathrm{L}$ ultrasound contrast agent. The optical image and IVUS image are from the same embryo and same region of interest showing corresponding vessel networks.

The chicken embryo and CAM vessels can also be used to investigate ultrasound-mediated drug delivery for which we show one example. Since the yolk obstructs the light path during imaging, the removal of the yolk sack is necessary to optically investigate drug delivery in the embryo and CAM vessels. For this study, the embryo and CAM were prepared for microscopic imaging as explained in section 2.2 by separating the membrane containing the embryo and CAM from the yolk sack (Figure 4C). In these embryos, the heart rate is stable around $80 \mathrm{bpm}$ and the embryos stay alive for up to $2 \mathrm{~h}$ when kept at a $37^{\circ} \mathrm{C}^{7}$. Figure 10 shows an ultrasound and microbubble mediated drug delivery study in endothelial cells of the CAM vessels. Lipid-coated microbubbles, targeted to the vessel wall using $\alpha_{v} \beta_{3}$-antibodies and stained with the fluorescent dye $\mathrm{Dil}^{7}$, were injected into the CAM vessels (Figure 10A,C). CAM vessel endothelial cell nuclei were stained with Hoechst 33342 (Figure 10B) and the model drug Propidium lodide $(\mathrm{PI})$ was used to visualize sonoporation ${ }^{7}$. Both these dyes were injected simultaneously with the microbubbles. Upon ultrasound treatment $(1 \mathrm{MHz}, 200 \mathrm{kPa}$ peak negative pressure, single burst of 1000 cycles), PI 
uptake was observed in the nuclei closest to the targeted microbubbles (Figure 10D). This shows that the ultrasound- induced oscillations of the targeted microbubbles were able to create a pore in the endothelial cell membrane.
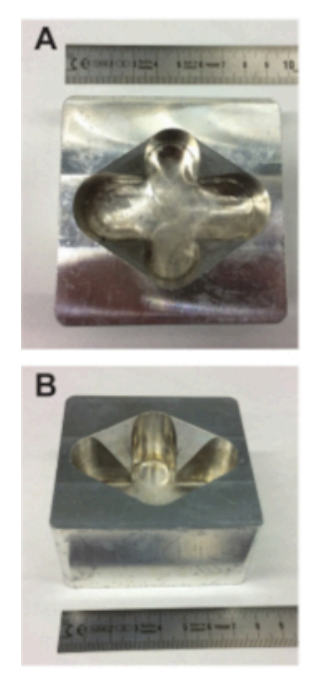

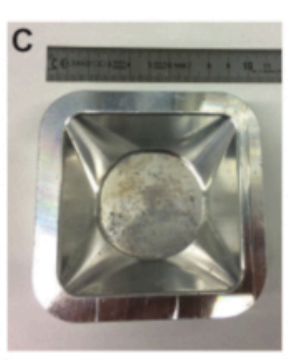

D

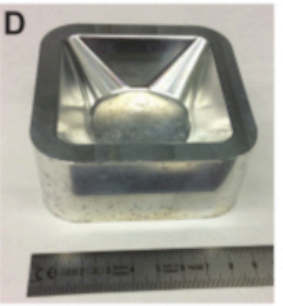

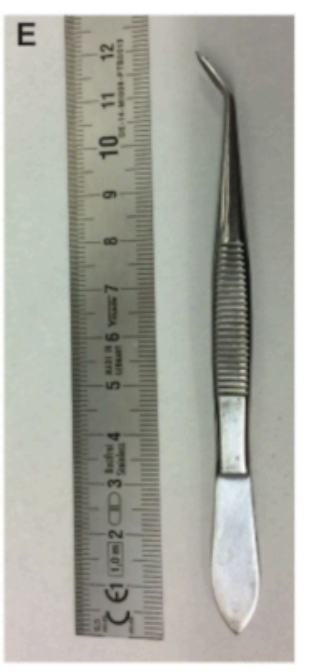

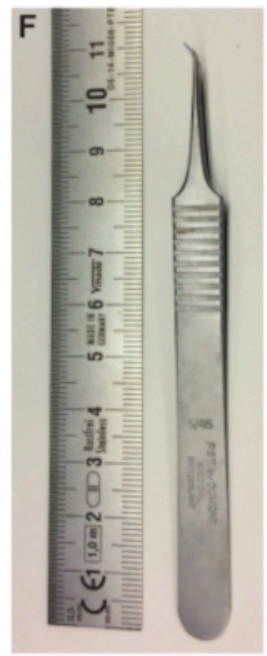

Figure 1. Embryo preparation equipment. (A-B) top and side view of the metal egg holder and (C-D) top and side view of the metal weighing boat holder. (E-F) tweezers needed to take the egg content out of the shell. Scale in $\mathrm{cm}$. Please click here to view a larger version of this figure. 

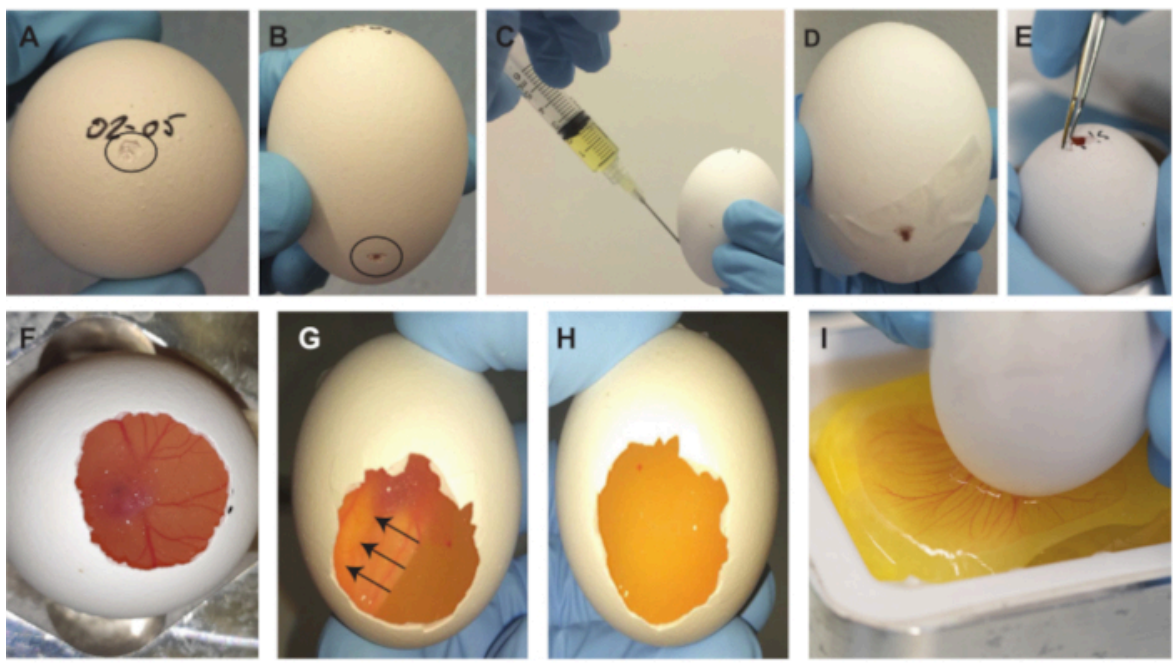

Figure 2. Embryo removal procedure. (A) Small indent on top of the egg, indicated by the black circle. (B) Small indent 2/3 down the egg, indicated by the black circle. (C) Withdrawing $\sim 2 \mathrm{~mL}$ of egg white. (D) Sealed gap on the side with tape. (E) Enlarging the small opening on the top of the egg. (F) The embryo becomes visible after removing part of the shell. (G-H) After rotating the egg $180^{\circ}$, the embryo floats up and will become invisible (arrows indicate moving direction of the embryo). After 1-2 min, the embryo is invisible from the bottom. (I) After scratching the membrane, the egg content drops into the weighing boat. Please click here to view a larger version of this figure. 

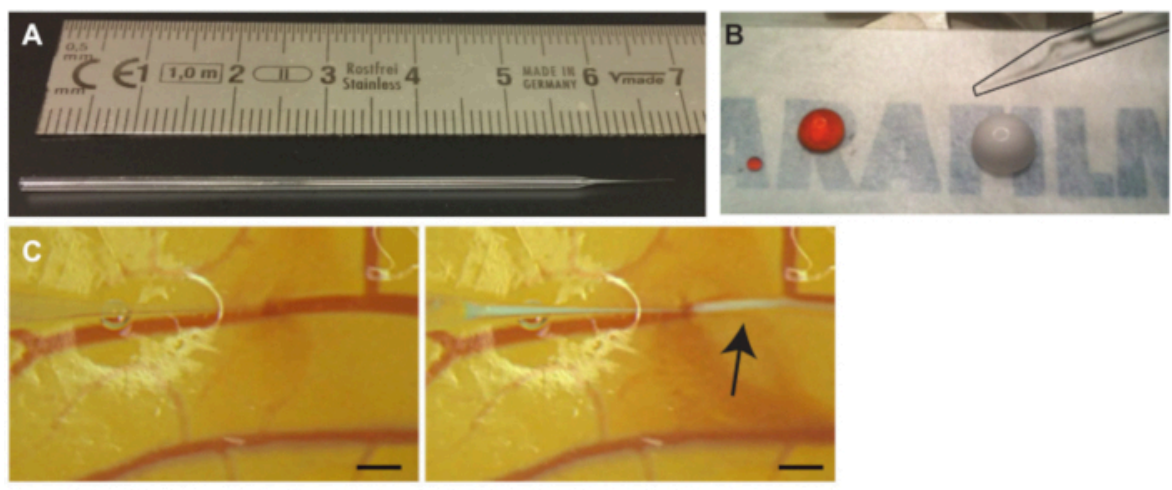

Figure 3. Injection of microbubbles into the CAM vessels. (A) Glass capillary needle. Scale in $\mathrm{cm}$. (B) Propidium iodide (PI) solution (left drop) and microbubbles (right drop) prior to aspiration before injection. Needle (outlined in black) can be seen in the top right corner (C) Microbubble injection. The capillary needle tip is positioned inside the lumen of one of the veins (left). Microbubbles, the white cloud indicated with an arrow, are injected and disperse following the blood stream (right). Scale bar represents $1 \mathrm{~mm}$. Please click here to view a larger version of this figure.
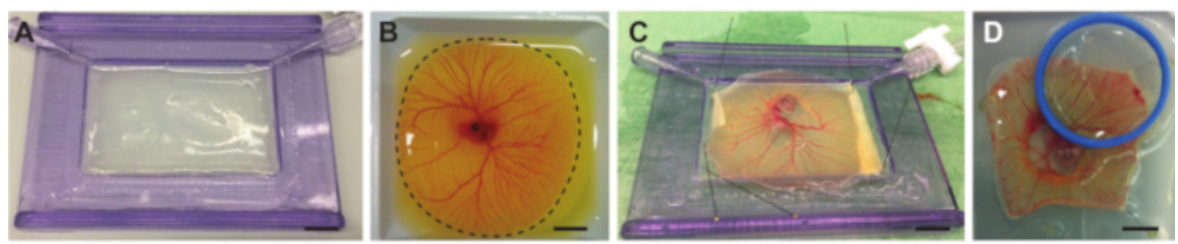

Figure 4. Removing embryo and CAM from yolk sack and placing on holder with acoustic membrane. (A) Holder with acoustic membrane filled with agarose layer. (B) Chicken embryo and CAM vessel in weighing boat before cutting. Dotted line indicates the cutting line around the CAM. (C) Chicken embryo and CAM separated from yolk and pinned down on acoustic membrane. (D) Pinned down chicken embryo with an acoustically and optically transparent membrane in a holder (blue) placed on top of the CAM. The holder can be filled with demi water so a water dipping objective can be used. All scale bars represent $1 \mathrm{~cm}$. Please click here to view a larger version of this figure. 

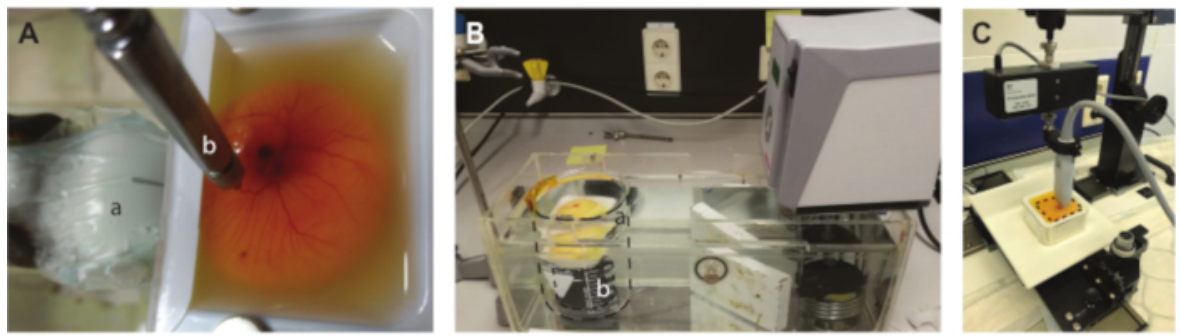

Figure 5. Different setups for chicken embryo and CAM ultrasound imaging. (A) Setup for ultrasound imaging from the side. Chicken embryo was placed in a custom-adjusted weighing boat with one acoustically transparent wall and placed in a $37^{\circ} \mathrm{C}$ water bath. The ultrasound transducer was positioned on the left (a) side next to the acoustically transparent wall and the laser (b) for photoacoustic imaging on top. (B) Setup for ultrasound imaging from the top. Embryo and CAM were submerged in a beaker of PBS that was placed in a $37^{\circ} \mathrm{C}$ water bath. Dashed outline shows the $2 \mathrm{~L}$ glass beaker (a) with the $500 \mathrm{~mL}$ glass beaker (b) inside. (C) Setup for ultrasound imaging from the top with a movable transducer. A thin agarose pad (dotted line) was placed on top of the embryo with a thin layer of PBS as coupling between the transducer and the agarose surface. Please click here to view a larger version of this figure.
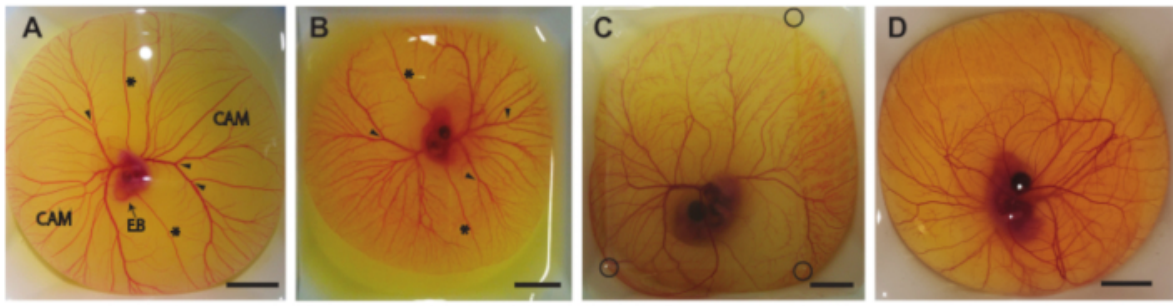

Figure 6. Egg content outside the shell. (A) Egg content taken out of the shell after 5 days of incubation. The chorioallantoic membrane (CAM), embryo body (EB), anterior and posterior vitelline veins $\left({ }^{*}\right)$, and appropriate sites for injection (arrowheads) are indicated. (B) Egg content taken out of the shell after 6 days of incubation. The anterior and posterior vitelline veins $\left({ }^{*}\right)$ and appropriate sites for injection (arrowheads) are indicated. (C) Egg content taken out of the shell after 7 days of incubation. A patch of agarose is placed on top to allow for ultrasound imaging. The corners of the agarose patch are indicated with black circles. (D) Egg content taken out of the shell after 8 days of incubation. All scale bars represent $1 \mathrm{~cm}$. Please click here to view a larger version of this figure. 

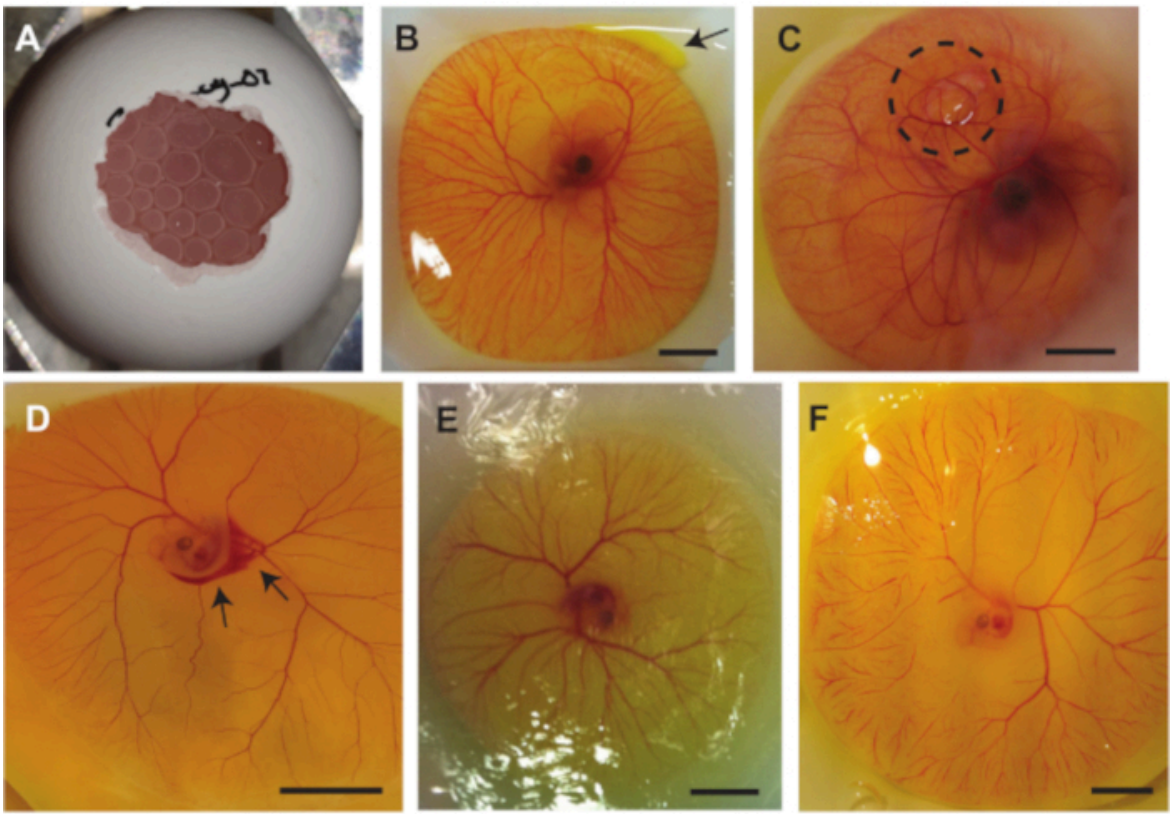

Figure 7. Complications which can occur during the procedures with the chicken embryo and CAM model. (A) Air bubbles trapped under the CAM when taking the egg content out of the shell using method 1.2 (5-day old embryo) or 1.3 (6 to 7-day old embryo). (B) Small leakage of yolk indicated with an arrow on the top right (6-day old embryo). (C) Air trapped under the CAM, indicated by the black dotted circle (7-day old embryo). (D) Bleeding, indicated with the black arrows (5-day old embryo. (E) Dried out embryo and CAM (5-day old embryo). (F) Massive capillary obstructions (5-day old embryo). All scale bars represent $1 \mathrm{~cm}$. Please click here to view a larger version of this figure. 


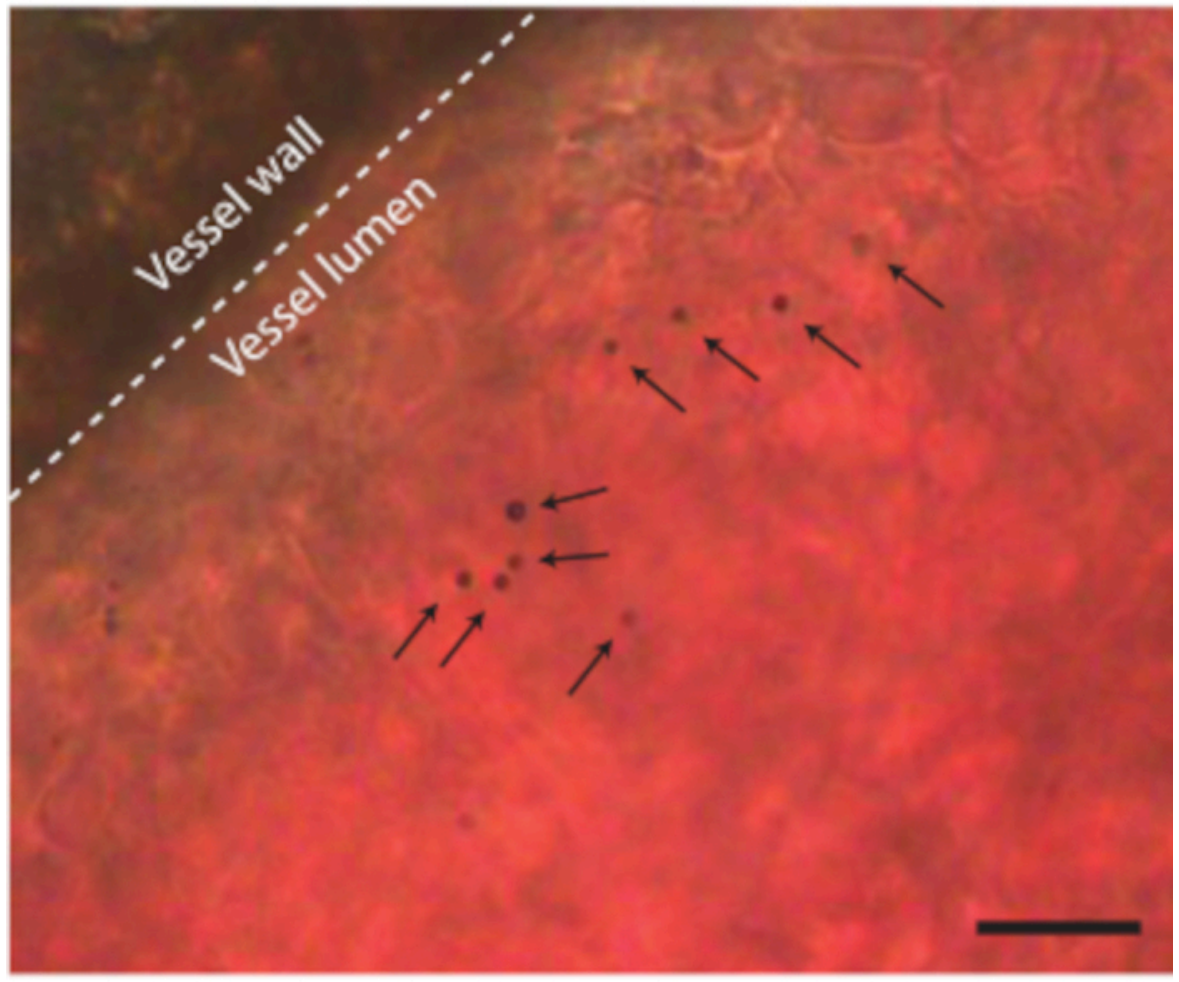

Figure 8. Microbubbles in CAM blood vessel. The vessel wall is indicated with a dotted line and single microbubbles are indicated with arrows. Scale bar represents $20 \mu \mathrm{m}$. The corresponding microscopy recording can be found in Supplemental Video 1. Please click here to view a larger version of this figure. 

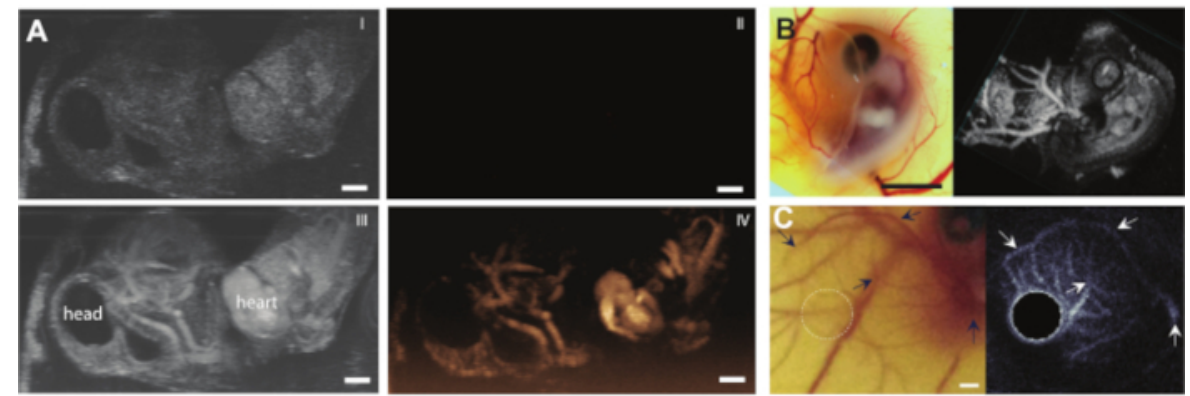

Figure 9. Contrast-enhanced ultrasound imaging in chicken embryos and CAM vessels. (A) Maximum-intensity projection of B-Mode (I, III) and real-time subharmonic (II, IV) images (preclinical animal ultrasound machine with MS250 probe, $30 \mathrm{MHz}$ transmitting and $15 \mathrm{MHz}$ receiving frequency, $10 \%$ power) of a 6-day old embryo with a patch of agarose on top. Top images (I, II) show results before and bottom (III, IV) after injection of $5 \mu \mathrm{L}$ ultrasound contrast agent. Scale bar represents $1 \mathrm{~mm}$. This image has been modified with permission from Daeichin et al. $2015^{11}$ (B) Optical (left) and 3D subharmonic imaging (right) of a 6-day old chicken embryo with a patch of agarose on top. CAM vessels were injected with 5 $\mu \mathrm{L}$ ultrasound contrast agent and imaging was performed with a high-frequency probe (preclinical animal ultrasound machine with MS550s probe, transmission frequency of $40 \mathrm{MHz}$, peak negative pressure $\sim 300 \mathrm{kPa}$, rendered in preclinical animal ultrasound machine 3-D mode). Scale bar represents $5 \mathrm{~mm}$. This image has been modified with permission from Daeichin et al. $2015^{11}$. (C) Optical image (left) and mean intensity projection of ultraharmonic intravascular ultrasound (IVUS) (right) of the CAM microvasculature of a 6 -day old embryo. CAM vessels were injected with $4 \pm 1 \mu \mathrm{L}$ contrast agent. Ultraharmonic IVUS imaging was performed with a custom IVUS probe (transmission frequency $35 \mathrm{MHz}$, peak negative pressure 600 $\mathrm{kPa}$ ). Both images are made from the same embryo and region of interest. Arrows indicate corresponding vessels in the two images. Scale bar represents $1 \mathrm{~mm}$. This image has been modified with permission from Maresca et al. $2014^{12}$. Please click here to view a larger version of this figure. 

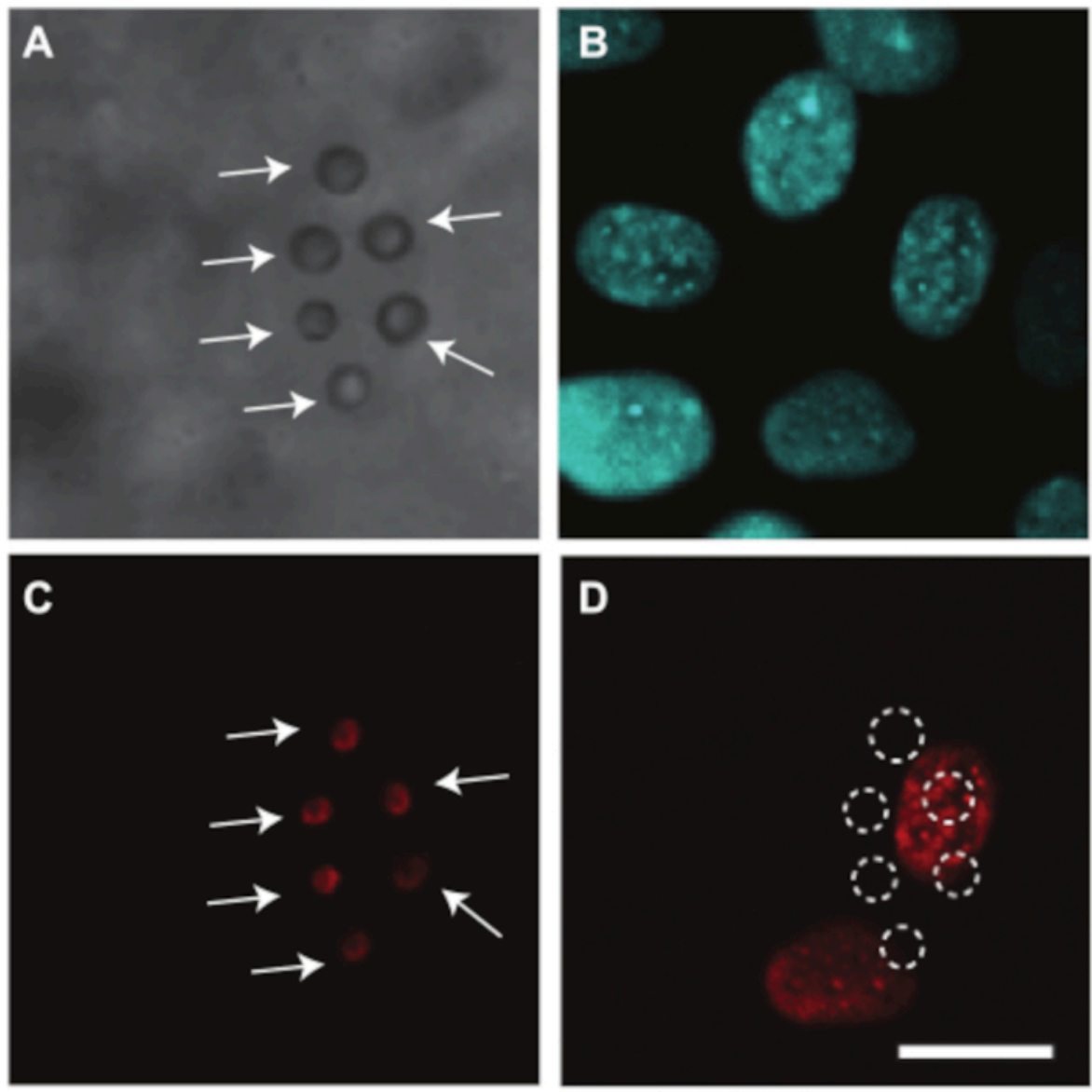

Figure 10. Drug delivery to CAM vessel endothelial cells in 6-day old embryo. (A) Brightfield image of six $\alpha_{\mathrm{V}} \beta_{3}$-targeted microbubbles, indicated with white arrows, adhering to the vessel wall before ultrasound treatment. (B) Endothelial cell nuclei fluorescently stained before ultrasound treatment. (C) Fluorescent image of the stained targeted microbubbles, indicated with white arrows, before ultrasound treatment. (D) Uptake of the model drug propidium iodide (PI) into the cell nuclei underneath the targeted microbubbles after ultrasound treatment (1 MHz, $200 \mathrm{kPa}$ peak negative pressure, single burst of $1000 \mathrm{cycles}$ ). Scale bar represents $10 \mu \mathrm{m}$ and applies to all images. This image has been modified with permission from Skachkov et al. $2014^{7}$. Please click here to view a larger version of this figure.

\section{SUPPLEMENTARY FILES}

Supplemental Video 1. Microbubbles in CAM blood vessel. Scale bar represents $20 \mu \mathrm{m}$. Please click here to download this video.

\section{Discussion}

This protocol describes three methods on how to obtain and use 5 to 8-day old chicken embryos and their CAM as an in vivo model to study contrast-enhanced ultrasound imaging and microbubble-mediated drug delivery. The most critical steps for taking 5-day old (section 1.2) and 6 to 7-day old 
(section 1.3) embryos out of the shell are: 1) make the small hole in the top of the egg to go through the entire eggshell into the air sac before withdrawing egg white; 2) create smooth edges for the large opening in the shell. For the method for taking 8-day old embryos out of the shell (section 1.4) the most critical steps are: 1) Make a sufficient number of indents to create a nice crack along the egg; 2) Keep the egg submerged in PBS. To ensure embryo viability for all methods it is important to keep the egg and its contents at $37^{\circ} \mathrm{C}$. In addition, avoid injecting into a CAM artery. Visually monitoring the heart rate of the embryo during the studies is recommended to ensure embryo vitality. To confirm the exact developmental stage of the embryo, the indication of Hamburger \& Hamilton ${ }^{22}$ can be used.

It is important to prevent damage to the embryo, CAM, and yolk sack. This damage can affect the viability, blood flow, and visibility of the embryo and CAM. In addition, damage to the yolk sack and consequently a low rigidity of the membrane makes an injection into the CAM vessels impossible. A 5day old embryo has a relatively small air sac so to be able to make a sufficiently large hole in the shell through which the egg content can be removed, $2 \mathrm{~mL}$ of egg white needs to be withdrawn. As a result, more space between the eggshell and embryo is created. After withdrawal of the egg white, a piece of tape needs to close off the hole where the needle went in. If egg white still leaks out, apply another piece of tape. Beside this, the application of tape on the hole on the side creates a vacuum inside the egg which prevents the egg content from falling out due to its own weight when the large hole is created in step 1.2.2.8. Damage to the embryo or CAM can also occur when the edge of the eggshell was too sharp or when the egg content is dropped into the weighing boat too rigorously, so the eggshell should be kept very close to the weighing boat. Between day 5 and 6 of development, the CAM starts to attach to the shell membrane ${ }^{32}$. This attachment increases the risk of damaging the embryo and CAM when taking the egg content out of the eggshell. By opening the egg after injection of PBS into it for a 6 to 7-day incubated egg or in a PBS-filled container as described for an 8-day incubated egg, the risk of damage is reduced. Regarding an injection into a CAM vein: if the first injection fails, a second injection can be done further upstream in the same vein if the damage was minor or in another CAM vein. Separation of the embryo and CAM from the yolk makes the embryo and CAM vessels optically transparent. As a consequence, the embryo loses its primary source of nutrients ${ }^{33}$. This loss of nutrients could be an explanation for the observed lower heart rate of 80 bpm in comparison to $\sim 190$ for a 6-day old embryo that is still in contact with the yolk ${ }^{30}$ and the reduced survival time of $2 \mathrm{~h}$ after this separation procedure. Another factor that can play a role in the reduced heart rate and survival time is the challenge to keep the yolk-separated embryo and CAM vessels at $37{ }^{\circ} \mathrm{C}$. A microscope stage incubator may be of aid. In addition to this, the detachment of the CAM from the yolk likely leads to mechanical changes in the tissue since the membrane tension becomes less. The lower membrane tension may cause an increased inner vessel shear rate which leads to a lower heart rate.

The ex ovo chicken embryo and CAM vessels have some limitations as in vivo model, including short time observations only, for contrast enhanced ultrasound imaging and microbubble-mediated drug delivery studies. Due to the small blood volume of $100 \pm 23 \mu \mathrm{L}$ at day 5 and $171 \pm 23 \mu \mathrm{L}$ at day $6^{34}$, a maximum volume of $\sim 5 \mu \mathrm{L}$ can be injected. In the later stages of development (day 7 and older), the vessel stiffness increases and the yolk elasticity decreases. This can complicate a successful injection in older embryos. Once the microbubbles are injected, they circulate for hours 
because the chicken embryo does not have a fully developed immune system at this stage ${ }^{35}$. Therefore, microbubbles are not cleared within $\sim 6$ min as in humans ${ }^{36,37}$ making typical ultrasound molecular imaging studies with a 5-10 min waiting period for non-bound targeted microbubbles to be cleared ${ }^{38}$ not feasible. In order to target microbubbles, suitable ligands able to bind to avian endothelial cells need to be used such as previously described for the angiogenesis marker $\alpha_{v} \beta_{3}{ }^{7}$. Other aspects to consider for this model are the increased difficulty of separating the embryo and CAM vessels from the yolk in older embryos (> 8 days) and lower hematocrit of $\sim 20 \%^{39}$ in comparison to humans. The latter may affect microbubbles oscillations because it is known that microbubble oscillations are damped in a more viscous environment ${ }^{40}$. CAM arteries are less oxygenated than CAM veins $^{41,42}$. This difference should be taken into account when for example studying photoacoustic imaging of blood oxygenation.

The methods described here allow the egg content to be taken out of the eggshell on the day of the ultrasound imaging or drug delivery study, typically at day 5 to 8 of incubation. This is different to existing methods where the egg content is taken out of the shell after a 3-day incubation and further developed as ex ovo culture $18,20,21$. The advantages are a higher survival rate of $90 \%$ for 5 -day, $75 \%$ for 6 -day, $50 \%$ for 7 -day, and $60 \%$ for 8 -day old incubated eggs in comparison to $~ 50 \%$ for 3-day old embryos taken out of the eggshell and further incubated ex ovo ${ }^{1,18}$ the avoidance of antibiotics during culture $^{18,20}$ and large sterile incubator for the ex ovo culture. The survival of the 6-to-8-day old embryos is lower because the CAM starts to attach itself to the shell ${ }^{21}$ which leaves the CAM membrane more prone to rupture upon extraction. The separation of the embryo with the CAM form the yolk is also described making the embryo and CAM optically transparent.

By placing the egg content in different setups, the chicken embryo and CAM can be used for a multitude of ultrasound imaging studies, like IVUS, photoacoustic, without or with ultrasound contrast agents in $2 \mathrm{D}$ and $3 \mathrm{D}$. The focus can be on developing new ultrasound pulsing schemes or testing out novel transducers. Besides this, the model can also be used to investigate novel ultrasound contrast agents and their behavior in blood vessels under flow. Since the mechanism of microbubble-mediated drug delivery is still unknown ${ }^{43}$, the use of the in vivo CAM model may help in elucidating the mechanism by studying the microbubble behavior in relation to the cellular response. Finally, the CAM vessels have proven to be a suitable system to investigate xenograft tumor transplantation ${ }^{44}$. This creates the possibility to use the CAM vessel as a model to investigate tumor imaging using ultrasound and to investigate the blood flow inside the tumor using CEUS. The tumors are typically grafted on the CAM vessels of 8 or 9-day old embryos ${ }^{1,14,45}$, for which the embryo is taken out of the eggshell at day 3 of incubation and further developed ex ovo. The methods described in this protocol could be used to grow embryos in ovo until the day of tumor grafting.

The authors trust that this paper will be helpful for researchers that want to use chicken embryos and their chorioallantoic membrane (CAM) as an in vivo model for applications of contrast agents and flow studies.

\section{Disclosures}

The authors have nothing to disclose.

\section{Acknowledgments}


This work was supported by the Applied and Engineering Sciences (TTW) (Vidi-project 17543), part of NWO. The authors would like to thank Robert Beurskens, Luxi Wei, and Reza Pakdaman Zangabad from the Department of Biomedical Engineering and Michiel Manten and Geert Springeling from the Department of Experimental Medical Instrumentation for technical assistance, all from the Erasmus MC University Medical Center Rotterdam, the Netherlands.

\section{References}

1. Martowicz, A., Kern, J., Gunsilius, E., Untergasser, G. Establishment of a Human Multiple Myeloma Xenograft Model in the Chicken to Study Tumor Growth, Invasion and Angiogenesis. Journal of Visualized Experiments. (99), 1-9 (2015).

2. Schmitz, M., Nelemans, B.K.A., Smit, T.H. A Submerged Filter Paper Sandwich for Long-term Ex ovo Time-lapse Imaging of Early Chick Embryos. Journal of Visualized Experiments. (118), 1-12 (2016).

3. Brauer, R., Chen, P. Influenza Virus Propagation in Embryonated Chicken Eggs. Journal of Visualized Experiments. (97), 1-6 (2015).

4. Oosterbaan, A.M., Ursem, N.T.C., Struijk, P.C., Bosch, J.G., van der Steen, A.F.W., Steegers, E.A.P. Doppler flow velocity waveforms in the embryonic chicken heart at developmental stages corresponding to 5-8 weeks of human gestation. Ultrasound in Obstetrics and Gynecology. 33(6), 638-644 (2009).

5. McQuinn, T.C., Bratoeva, M., DeAlmeida, A., Remond, M., Thompson, R.P., Sedmera, D. High-Frequency Ultrasonographic Imaging of Avian Cardiovascular Development. Developmental Dynamics. 236(12), 3503-3513 (2007)
6. Stieger, S.M., Caskey, C.F., Adamson, R.H., Curry, F.E., Wisner, E.R., Ferrara, K.W. Enhancement of Vascular Permeability with Low-Frequency Contrast-enhanced Ultrasound in the Chorioallantoic Membrane Model. Radiology. 243(1), 112-121 (2007).

7. Skachkov, I., Luan, Y., van der Steen, A.F.W., De Jong, N., Kooiman, K. Targeted microbubble mediated sonoporation of endothelial cells in vivo. IEEE Transactions on Ultrasonics, Ferroelectrics, and Frequency Control. 61(10), 1661-1667 (2014).

8. Vargas, A., Zeisser-Labouèbe, M., Lange, N., Gurny, R., Delie, F. The chick embryo and its chorioallantoic membrane (CAM) for the in vivo evaluation of drug delivery systems. Advanced Drug Delivery Reviews. 59(11), 1162-1176 (2007).

9. Rytelewski, M., Buensuceso, A., Leong, H.S., Deroo, B.J., Chambers, A.F., Koropatnick, J. Evaluating the Effectiveness of Cancer Drug Sensitization In Vitro and In vivo. Journal of Visualized Experiments. (96), 1-5 (2015).

10. Schmitd, L.B., Liu, M., Scanlon, C.S., Banerjee, R., Silva, N.J.D. The Chick Chorioallantoic Membrane In vivo Model to Assess Perineural Invasion in Head and Neck Cancer. Journal of Visualized Experiments. (148), 1-10 (2019).

11. Daeichin, V., Bosch, J.G., Needles, A., Foster, F.S., van der Steen, A., de Jong, N. Subharmonic, non-linear fundamental and ultraharmonic imaging of microbubble contrast at high frequencies. Ultrasound in Medicine and Biology. 41(2), 486-497 (2015).

12. Maresca, D. et al. Imaging microvasculature with contrast-enhanced ultraharmonic ultrasound. Ultrasound in Medicine and Biology. 40(6), 1318-1328 (2014). 
13. Lindsey, B.D. et al. High Resolution Ultrasound Superharmonic Perfusion Imaging: In vivo Feasibility and Quantification of Dynamic Contrast-Enhanced Acoustic Angiography. Annals of Biomedical Engineering. 45(4), 939-948 (2017).

14. Paproski, R.J., Jovel, J., Wong, G.K.S., Lewis, J.D., Zemp, R.J. Enhanced detection of cancer biomarkers in blood-borne extracellular vesicles using nanodroplets and focused ultrasound. Cancer Research. 77(1), 3-13 (2017).

15. Huang, C. et al. Short Acquisition Time Super-Resolution Ultrasound Microvessel Imaging via Microbubble Separation. Scientific Reports. 10, 1-13 (2020).

16. Lowerison, M.R., Huang, C., Kim, Y., Lucien, F., Chen, S., Song, P. In vivo Confocal Imaging of Fluorescently Labeled Microbubbles: Implications for Ultrasound Localization Microscopy. IEEE Transactions on Ultrasonics, Ferroelectrics, and Frequency Control. 67(9), 1811-1819 (2020).

17. Faez, T., Skachkov, I., Versluis, M., Kooiman, K., de Jong, N. In vivo Characterization of Ultrasound Contrast Agents: Microbubble Spectroscopy in a Chicken Embryo. Ultrasound in Medicine and Biology. 38(9), 1608-1617 (2012).

18. Yalcin, H.C., Shekhar, A., Rane, A.A., Butcher, J.T. An ex-ovo Chicken Embryo Culture System Suitable for Imaging and Microsurgery Applications. Journal of Visualized Experiments. (44), 4-7 (2010).

19. Kokhuis, T.J.A. et al. Intravital microscopy of localized stem cell delivery using microbubbles and acoustic radiation force. Biotechnology and Bioengineering. 112(1), 220-227 (2015).
20. Cloney, K., Franz-Odendaal, T.A. Optimized Ex-ovo Culturing of Chick Embryos to Advanced Stages of Development. Journal of Visualized Experiments. (95), 6-10 (2015).

21. Dohle, D.S. et al. Chick ex ovo Culture and ex ovo CAM Assay: How it Really Works. Journal of Visualized Experiments. (33), 2-7 (2010).

22. Hamburger, V., Hamilton, H. A Series Of Normal Stages In The Developent Of The Chick Embryo. Journal of Morphology. 88(1), 231-272 (1951).

23. Ribatti, D. A morphometric study of the expansion of the chick vasculosa in shell-less culture. Journal of Anatomy. 186, 639-644 (1995).

24. Ribatti, D., Nico, B., Vacca, A., Roncali, L., Burri, P.H., Djonov, V. Chorioallantoic Membrane Capillary Bed: A Useful Target for Studying Angiogenesis and AntiAngiogenesis In vivo. Anatomical Record. 324, 317-324 (2001).

25. DeFouw, D.O., Rizzo, V.J., Steinfeld, R., Feinberg, R.N. Mapping of the Microcirculation in the Chick Chorioallantoic Membrane during Normal Angiogenesis. Microvascular research. 38, 136-147 (1989).

26. Beekers, I., van Rooij, T., van der Steen, A.F.W., de Jong, N., Verweij, M.D., Kooiman, K. Acoustic characterization of the CLINIcell for ultrasound contrast agent studies. IEEE Transactions on Ultrasonics, Ferroelectrics, and Frequency Control. 66(1), 244-246 (2019).

27. Lang, E.R., Rha, C. Apparent shear viscosity of native egg white. Journal of Food Science and Technology. 17, 595-606 (1982). 
28. Daeichin, V. et al. Microbubble Composition and Preparation for Imaging: In Vitro and In vivo Evaluation. IEEE TRANSACTIONS ON ULTRASONICS. 64(3), 555-567 (2017).

29. Mcferrin, H.E., Olson, S.D., Gutschow, M. V, Semon, J.A., Sullivan, D.E., Prockop, D.J. Rapidly Self-Renewing Human Multipotent Marrow Stromal Cells (hMSC) Express Sialyl Lewis $X$ and Actively Adhere to Arterial Endothelium in a Chick Embryo Model System. PLoS ONE. 9(8), 1-11 (2014).

30. Akiyama, R., Mitsubayashi, H., Tazawa, H., Burggren, W.W. Heart rate responses to altered ambient oxygen in early (days 3-9) chick embryos in the intact egg. Journal of Comparative Physiology - B Biochemical, Systemic, and Environmental Physiology. 169(2), 85-92 (1999).

31. Stuart Foster, F., Hossack, J., Lee Adamson, S. Microultrasound for preclinical imaging. Interface Focus. 1, 576-601 (2011).

32. Gabrielli, M.G., Accili, D. The Chick Chorioallantoic Membrane: A Model of Molecular, Structural, and Functional Adaptation to Transepithelial Ion Transport and Barrier Function during Embryonic Development. Journal of Biomedicine and Biotechnology. 1-12 (2010).

33. van der Wagt, I., de Jong, I.C., Mitchell, M.A., Molenaar, R., van den Brand, H. A review on yolk sac utilization in poultry. Poultry Science. 99, 2162-2175 (2020).

34. Kind, C. The development of the circulating blood volume of the chick embryo. Anatomy and Embryology. 147, 127-132 (1975).

35. Ribatti, D. Chick Embryo Chorioallantoic Membrane as a Useful Tool to Study Angiogenesis. International Review of Cell and Molecular Biology. 270, 181-224 (2008).
36. Schneider, M. Characteristics of SonoVue(TM). Echocardiography. 16(7), 743-746 (1999).

37. Kitzman, D.W., Goldman, M.E., Gillam, L.D., Cohen, J.L., Aurigemma, G.P., Gottdiener, J.S. Efficacy and Safety of the Novel Ultrasound Contrast Agent Perflutren (Definity) in Patients With Suboptimal Baseline Left Ventricular Echocardiographic Images. American Journal of Cardiology. 86, 669-674 (2000).

38. Kosareva, A., Abou-Elkacem, L., Chowdhury, S., Lindner, J.R., Kaufmann, B.A. Seeing the InvisibleUltrasound Molecular Imaging. Ultrasound in Medicine and Biology. 46(3), 479-497 (2020).

39. Al-Roubaie, S., Jahnsen, E.D., Mohammed, M., Henderson-Toth, C., Jones, E.A.V. Rheology of embryonic avian blood. American Journal of Physiology - Heart and Circulatory Physiology. 301(6), 2473-2481 (2011).

40. Helfield, B., Chen, X., Qin, B., Villanueva, F.S. Individual lipid encapsulated microbubble radial oscillations: Effects of fluid viscosity. The Journal of the Acoustical Society of America. 139(1), 204-214 (2016).

41. Metcalfe, J., Stock, M.K. Oxygen exchange in the chorioallantoic membrane, avian homologue of the mammalian placenta. Placenta. 14, 605-613 (1993).

42. Tazawa, H. Oxygen and CO2 exchange and acid-base regulation in the avian embryo. American Journal of Zoology. 20, 395-404 (1980).

43. Kooiman, K. et al. Ultrasound-Responsive Cavitation Nuclei for Therapy and Drug Delivery. Ultrasound in Medicine and Biology. 46(6), 1296-1325 (2020).

44. Li, M., Pathak, R.R., Lopez-rivera, E., Friedman, S.L., Aguirre-ghiso, J.A., Sikora, A.G. The In ovo Chick 
Chorioallantoic Membrane (CAM) Assay as an Efficient Xenograft Model of Hepatocellular Carcinoma. Journal of Visualized Experiments. 104, 1-6 (2015).

45. Sys, G.M.L. et al. The In ovo CAM-assay as a Xenograft Model for Sarcoma. Journal of Visualized Experiments.

77, 1-7 (2013). 